Analyse fonctionnelle/Functional analysis

\title{
Amenable groups and measure concentration on spheres $\mathrm{\theta}$
}

\author{
Vladimir PESTOV
}

School of Mathematical and Computing Sciences, Victoria University of Wellington, P.O. Box 600, Wellington, New Zealand.

E-mail: vova@mcs.vuw.ac.nz

Home: http://www.vuw.ac.nz/ vova/

\begin{abstract}
It is proved that a discrete group $G$ is amenable if and only if for every unitary representation of $G$ in an infinite-dimensional Hilbert space $\mathcal{H}$ the maximal uniform compactification of the unit sphere $\mathbb{S}_{\mathcal{H}}$ has a $G$-fixed point, that is, the pair $\left(\mathbb{S}_{\mathcal{H}}, G\right)$ has the concentration property in the sense of Milman. Consequently, the maximal $U(\mathcal{H})$ equivariant compactification of the sphere in a Hilbert space $\mathcal{H}$ has no fixed points, which answers a 1987 question by Milman.
\end{abstract}

\section{Groupes moyennables et concentration de mesure sur les sphères}

Résumé - On démontre qu'un groupe discret $G$ est moyennable si et seulement si, pour toute représentation unitaire de $G$ dans un espace de Hilbert $\mathcal{H}$ de dimension infinie, it existe un point fixe de $G$ dans le compactifié de Samuel de la sphère $\mathbb{S}_{\mathcal{H}}$, c'est-à-dire la paire $\left(\mathbb{S}_{\mathcal{H}}, G\right)$ possède la propriété de concentration au sens de Milman. Par conséquent, le compactifié maximal $U(\mathcal{H})$-équivariant de la sphère unité d'un espace de Hilbert $\mathcal{H}$ ne contient aucun point fixe. Ceci permet de répondre à une question de Milman.

Version française abrégée. - Le phénomène de concentration de la mesure sur les structures de grande dimension [11, 16] été utilisé par M. Gromov et V.D. Milman dans [6, 10] pour établir un nombre de nouveaux théorèmes du type point fixe.

Soit $X=\left(X, \mathcal{U}_{X}\right)$ un espace uniforme et soit $F$ une famille des applications uniformément continues de $X$ dans lui-même. D'après Milman [10, 11], on dit que la paire $(X, F)$ possède la propriété de concentration si tout couvert fini de $X$ contient un élément $A$ tel que, pour tout $V \in \mathcal{U}_{X}$ et toute famille finie $f_{1}, f_{2}, \ldots, f_{n} \in F$, $n \in \mathbb{N}$, on a $\cap_{i=1}^{n} f_{i} V[A] \neq \emptyset$. Dans ce cas, il existe un point fixe par $F$ dans chaque compactifié $F$-équivariant de $X$. Gromov et Milman prouvent dans [6] que la paire $\left(\mathbb{S}_{\mathcal{H}}, G\right)$ possède la propriété de concentration, où $\mathbb{S}_{\mathcal{H}}$ est la sphère unité dans un

\footnotetext{
${ }^{\ddagger}$ Preprinted as Research Report 98-27, School of Mathematical and Computing Sciences, Victoria University of Wellington, October 1998. This is a version as of November 19, 1998, incorporating some revisions.
} 
espace de Hilbert $\mathcal{H}$ de dimension infinie, et $G=U(\infty):=\cup_{i=1}^{\infty} U(n)$ ou $G$ est un sous-groupe abélien de $U(\mathcal{H})$. Milman a posé dans [10, 11 la question naturelle suivante: est-ce que la paire $\left(\mathbb{S}_{\mathcal{H}}, U(\mathcal{H})\right)$ possède la propriété de concentration, où $U(\mathcal{H})$ dśigne le groupe de tous les opérateurs unitaires sur un espace de Hilbert $\mathcal{H}$ de dimension infinie?

L'objet de cette Note est d'établir un rapport entre la propriété de concentration et la moyennabilité des groupes et de répondre à cette question par la négative.

Pour un espace uniforme $X, C_{u}^{b}(X)$ désigne l'espace de Banach des fonctions uniformément continues bornées de $X$ dans $\mathbb{C}$ et $\sigma X$ désigne le compactifié de Samuel de $X$ [3], c'est-à-dire le spectre de Gelfand de la $C^{*}$-algèbre commutative $C_{u}^{b}(X)$.

Proposition A. - Pour un espace uniforme $X$ et une famille $F$ d'isomorphismes uniformes de $X$, les quatre assertions suivantes sont équivalentes:

1. La paire $(X, F)$ possède la propriété de concentration.

2. La paire $(\sigma X, F)$ possède la propriété de concentration.

3. Il existe un point fixe de $F$ dans $\sigma X$.

4. Il existe une moyenne multiplicative $F$-invariante $\operatorname{sur} C_{u}^{b}(X)$.

La question ci-dessus trouve alors sa réponse dans le résultat suivant.

Proposition B. - Un groupe localement compact $G$ est moyennable si et seulement si la paire $\left(\mathbb{S}_{2}, G\right)$ possède la propriété de concentration, où $\mathbb{S}_{2}$ dénote la sphère unité dans l'espace $L_{2}(G)$ de la représentation régulière gauche de $G$.

Corollaire $\mathbf{1}^{\circ}$ — Soit $\mathcal{H}$ un espace de Hilbert. Alors la paire $\left(\mathbb{S}_{\mathcal{H}}, U(\mathcal{H})\right)$ ne possède pas la propriété de concentration.

Exemple. - Soit $F_{2}$ le groupe libre à deux générateurs $a$ et $b$. Pour tout entier $n$, soit $W_{n}$ l'ensemble des $x \in F_{2}$ dont l'écriture en mot réduit commence par $a^{n}$. Soit $\mathcal{H}=$ $L_{2}\left(F_{2}\right)$. Posons $A_{1}=\left\{t \in \mathbb{S}:\left\|\chi_{W_{0}} \cdot t\right\| \leq 1 / 3\right\}, A_{2}=\left\{t \in \mathbb{S}:\left\|\chi_{W_{0}} \cdot t\right\| \geq 1 / 3\right\}$, et $F=\left\{a, a^{2}, a^{3}, a^{4}, b\right\}$. Alors $\mathbb{S}=A_{1} \cup A_{2}$, et on vérifie que $\mathcal{O}_{1 / 12}\left(A_{1}\right) \cap \mathcal{O}_{1 / 12}\left(b A_{1}\right)=\emptyset$ et $\mathcal{O}_{1 / 12}\left(A_{2}\right) \cap \cap_{i=1}^{4} \mathcal{O}_{1 / 12}\left(a^{i} A_{2}\right)=\emptyset$.

Nous notons $U(\mathcal{H})_{u}$ le groupe unitaire de $\mathcal{H}$ muni de la topologie normique.

Corollaires. $-\mathbf{2}^{\circ}$ Soit $\mathcal{H}$ un espace de Hilbert. Alors le compactifié maximal $U(\mathcal{H})_{u}$-équivariante [18] de la sphère unité $\mathbb{S}_{\mathcal{H}}$ ne contient aucun point fixe.

$3^{\circ}$ Le groupe de Calkin projectif opère continûment sur un espace compact $X$ de manière effective et minimale (c'est-à-dire l'orbite de chaque point est dense dans $X)$.

Pour les groupes discrets, nous allons obtenir le résultat plus fort que la proposition (B) qui suit.

Théorème. - Un groupe discret $G$ est moyennable si et seulement si, pour toute représentation unitaire de $G$ dans un espace de Hilbert $\mathcal{H}$ de dimension infinie, la paire $\left(\mathbb{S}_{\mathcal{H}}, G\right)$ possède la propriété de concentration.

La démonstration repose sur le lemme suivant. 
Lemme. - Soient $\epsilon>0$ et $C>0$. Pour toute suite $\left(k_{n}\right)_{n=1}^{\infty}$ d'entiers $\geq 1$ telle que $k_{n}=o(n)$, la mesure normalisée du $\epsilon$-voisinage de $\mathbb{S}^{n}$ dans $\mathbb{S}^{n+k_{n}}$ est minorée par $\exp (-C n)$ pour $n$ assez grand.

§0. Introduction. One of many ways in which the phenomenon of concentration of measure on high-dimensional structures [11, 16] manifests itself is via fixed point theorems of the type discovered by Gromov and Milman [6, 10].

Let $X=\left(X, \mathcal{U}_{X}\right)$ be a uniform space, and let $F$ be a family of uniformly continuous self-maps of $X$. Following Milman [10, 11], we say that the pair $(X, F)$ has the property of concentration if every finite cover $\gamma$ of $X$ contains an $A$ such that for every $V \in \mathcal{U}_{X}$ and every finite collection $f_{1}, f_{2}, \ldots, f_{n} \in F, n \in \mathbb{N}$, one has $\cap_{i=1}^{n} f_{i} V[A] \neq \emptyset$, where $V[A]$ is the $V$-neighbourhood of $A$. (Such an $A$ is called essential.)

The property of concentration implies (and, if $F$ is a group, is equivalent to, cf. Prop. 1.1 below) the existence of a common fixed point for $F$ in every $F$-equivariant uniform compactification of $X$. Among the results proved by Gromov and Milman [6, 10 are the following two.

(1) The pair $\left(\mathbb{S}^{\infty}, U(\infty)\right)$, where $\mathbb{S}^{\infty}$ is the unit sphere of $l_{2}$ (with the Euclidean distance and uniformity) and $U(\infty)=\cup_{i=1}^{\infty} U(n)$, has the property of concentration.

(2) If $A$ is an abelian subgroup of the full unitary group $U(\mathcal{H})$, then the pair $\left(\mathbb{S}_{\mathcal{H}}, A\right)$ has the property of concentration.

These results lead naturally to the following question, stated by Milman in 10 , 11]: does the pair $\left(\mathbb{S}_{\mathcal{H}}, U(\mathcal{H})\right)$ have the property of concentration for an infinitedimensional Hilbert space $\mathcal{H}$ ?

In this Note we give a negative answer (Corol. 2.3, Ex. 2.4). We obtain a new description of amenable groups in terms of the property of concentration of unit spheres in spaces of representations (Prop. 2.1 and Th. 2.6). Also, we deduce a number of dynamical corollaries.

$\S 1$. Fixed points and property of concentration. The Samuel compactification [3] of a uniform space $X=\left(X, \mathcal{U}_{X}\right)$ is a Hausdorff compact space $\sigma X$ together with a uniformly continuous mapping $i_{X}: X \rightarrow \sigma X$ such that every uniformly continuous mapping of $X$ to an arbitrary compact Hausdorff space factors through $i_{X}$. Notice that every uniformly continuous $f: X \rightarrow X$ determines a unique continuous mapping $\bar{f}: \sigma X \rightarrow \sigma X$. By $C_{u}^{b}(X) \cong C(\sigma X)$ we will denote the Banach space (moreover, commutative $C^{*}$-algebra) of all bounded uniformly continuous complexvalued functions on a uniform space $X$.

Proposition 1.1. For a family $F$ of isomorphisms of a uniform space $\left(X, \mathcal{U}_{X}\right)$, the following are equivalent.

1. The pair $(X, F)$ has the property of concentration.

2. The pair $(\sigma X, F)$ has the property of concentration.

3. The family $F$ has a common fixed point in the Samuel compactification of $X$.

4. There exists an F-invariant multiplicative mean on the space $C_{u}^{b}(X)$.

If $F$ is a family of uniformly continuous self-maps of $X$, (11) $\Rightarrow$ (2) $\Leftrightarrow$ (3) $\Leftrightarrow$ (田). 
Proof. (11) $\Rightarrow$ (2): trivial. (2) $\Rightarrow$ (3): emulates the proofs making up Sect. 4 in [10]. (3) $\Leftrightarrow$ (田): the Gelfand space of the commutative $C^{*}$-algebra $C_{u}^{b}(X)$ is $\sigma X$, and fixed points of $\sigma X$ correspond to $F$-invariant multiplicative means. (3) $\Rightarrow(\mathbb{1})$ : If $\gamma$ is a finite cover of $X$, then for some $A \in \gamma$ the closure of $i_{X}(A)$ in $\sigma X$ contains an $F$-fixed point. Such an $A$ is essential, which follows easily from the simple fact of general topology: if $B, C \subseteq X$ are such that for some $V \in \mathcal{U}_{X}, V[B] \cap V[B]=\emptyset$, then $\mathrm{cl}_{\sigma X}(B) \cap \mathrm{cl}{ }_{\sigma X}(C)=\emptyset$.

Remark 1.2. As pointed out in [4] on a similar occasion, the condition of uniform equicontinuity of $F$, imposed in [6, 10, 11], is superfluous.

\section{$\S 2$. Amenability, property of concentration, and Milman's question.}

Proposition 2.1. For a locally compact group $G$ the following are equivalent.

1. $G$ is amenable.

2. There exists a left $G$-invariant mean on the space $C_{u}^{b}\left(\mathbb{S}_{2}\right)$, where $\mathbb{S}_{2}$ is the unit sphere in $L_{2}(G)$.

3. The pair $\left(\mathbb{S}_{2}, G\right)$ has the concentration property.

Proof. (11) $\Rightarrow$ (3): According to Property $\left(\mathrm{P}_{2}\right)$ (see e.g. [12], Th. 4.4), for each $\epsilon>0$ and a compact $K \subseteq G$ there is an $f_{K, \epsilon} \in \mathbb{S}_{2}$ with $\left\|g f_{K, \epsilon}-f_{K, \epsilon}\right\|<\epsilon$ for all $g \in K$, where $(g f)(h)=f(g h)$. Consequently, the compact sets $\Phi_{K, V}=\{x \in$ $\sigma \mathbb{S}_{2}$ : for all $\left.g \in K,(x, g x) \in V\right\}$ are non-empty for each compact $K \subseteq G$ and every closed neighbourhood of the diagonal $V \in \mathcal{U}_{\sigma \mathbb{S}_{2}}$. Finally, since $\Phi_{K_{1}, V_{1}} \subseteq \Phi_{K_{2}, V_{2}}$ whenever $K_{1} \supseteq K_{2}$ and $V_{1} \subseteq V_{2}$, the intersection $\Phi=\cap_{K, V} F_{K, V}$ (consisting of all $G$-fixed points of $\left.\sigma \mathbb{S}_{2}\right)$ is non-empty.

(3) $\Rightarrow$ (2): use Prop. 1.1, (11) $\Rightarrow$ (4).

(2) $\Rightarrow$ (1) : Let $\phi$ be a left $G$-invariant mean on $C_{u}^{b}\left(\mathbb{S}_{2}\right)$. For every Borel set $A \subseteq G$ and each $f \in \mathbb{S}_{2}$ set $z_{A}(f)=\left\|\chi_{A} \cdot f\right\|^{2}$. Since the mapping $f \mapsto\|f\|^{2}$ is 2-Lipschitz on the unit sphere, so is the function $z_{A}: \mathbb{S}_{2} \rightarrow \mathbb{R}$, and $z_{A} \in C_{u}^{b}\left(\mathbb{S}_{2}\right)$. Set $m(A)=$ $\phi\left(z_{A}\right)$. Then $m$ is a finitely-additive left-invariant normalized measure on Borel subsets of $G$, vanishing on locally null sets, and consequently $G$ is amenable.

Corollary 2.2. The unit sphere of an infinite-dimensional Hilbert space $\mathcal{H}$ admits no left invariant means on bounded uniformly continuous functions with respect to the full unitary group $U(\mathcal{H})$.

Corollary 2.3. Let $\mathcal{H}$ be a Hilbert space. The pair $\left(\mathbb{S}_{\mathcal{H}}, U(\mathcal{H})\right)$ does not have the concentration property.

Combining the proof of 2.1 with von Neumann's proof of non-amenability of $F_{2}$ ([12], ex. 0.6), we obtain an explicit counter-example to Milman's question.

Example 2.4. Let $a, b$ be free generators of $F_{2}$, and let $\pi=\pi_{2}$ be the left regular representation of $F_{2}$ in $\mathcal{H}=l_{2}\left(F_{2}\right)$; we will write $x f$ for $\pi_{x}(f)$. Denote by $W_{n}$ the collection of all words whose irreducible representation starts with $a^{n}, n \in \mathbb{Z}$. Set $A_{1}=\left\{f \in \mathbb{S}:\left\|\chi_{W_{0}} \cdot f\right\| \leq 1 / 3\right\}, A_{2}=\left\{f \in \mathbb{S}:\left\|\chi_{W_{0}} \cdot f\right\| \geq 1 / 3\right\}$, and $F=$ $\left\{a, a^{2}, a^{3}, a^{4}, b\right\}$. Clearly, $\mathbb{S}=A_{1} \cup A_{2}$. Both $A_{1}$ and $A_{2}$ are $F$-inessential. Indeed, 
if $f \in A_{1}$, then $\left\|\chi_{W_{0}} \cdot b f\right\| \geq\left\|\chi_{F_{2} \backslash W_{0}} \cdot f\right\| \geq 2 / 3$ and consequently $\mathcal{O}_{1 / 12}\left(A_{1}\right) \cap$ $\mathcal{O}_{1 / 12}\left(b A_{1}\right)=\emptyset$. If $f \in A_{2}$, there is an $i \in\{1,2,3,4\}$ such that $\left\|\chi_{W_{-i}} \cdot f\right\|<1 / 6$, and consequently $\left\|\chi_{W_{0}} \cdot a^{i} f\right\|<1 / 6$, meaning that $\mathcal{O}_{1 / 12}\left(A_{2}\right) \cap \cap_{i=1}^{4} \mathcal{O}_{1 / 12}\left(a^{i} A_{2}\right)=$ $\emptyset$.

For discrete groups we are able to strengthen Proposition 2.1 significantly. The following is derived by recurrently applying Lévy's property of measure concentration on spheres to a nested sequence of neighbourhoods of $\mathbb{S}^{n}$ in $\mathbb{S}^{n+j}, j=1, \ldots, k_{n}$.

Lemma 2.5. Let $\epsilon>0$. The normalised rotation-invariant measure of the $\epsilon$ neighbourhood of $\mathbb{S}^{n}$ in $\mathbb{S}^{n+k_{n}}$ is asymptotically greater than any function $\exp (-C n)$, $C>0$, provided that $k_{n} / n \rightarrow 0$ as $n \rightarrow \infty$.

Theorem 2.6. A discrete group $G$ is amenable if and only if for every unitary representation $\pi$ of $G$ in an infinite-dimensional Hilbert space $\mathcal{H}_{\pi}$ the pair $\left(\mathbb{S}_{\pi}, G\right)$ has the concentration property.

Proof. $\Leftarrow$ : follows from Prop. 2.1. $\Rightarrow$ : let $\gamma$ be a finite cover of $\mathbb{S}_{\pi}$. It suffices to find an $F$-essential set $A \in \gamma$ for every given finite $F \subseteq G$. Choose $D=$ $\left\{g_{1}, g_{2}, \ldots, g_{d}\right\} \subseteq G$ so that $F=\left\{g_{j} g_{j-1} \cdots g_{2} g_{1}: j=1,2, \ldots, d\right\}$. Use the Følner condition applied to $D$ in order to construct an increasing chain of vector spaces $V_{n} \subset \mathcal{H}_{\pi}$ of finite dimension $i_{n}=\operatorname{dim} V_{n} \geq n$ such that $\pi_{g} \cdot V_{n} \subseteq V_{n+1}$ for all $g \in D$ and $i_{n+1} / i_{n} \rightarrow 1$ as $n \rightarrow \infty$. Denote $\mathbb{S}_{n}=\mathbb{S} \cap V_{n}$. Let $m=|\gamma|$. At least one $A \in \gamma$ must have the property that for each $n \in \mathbb{N}$ one can find $d+1$ natural numbers $n \leq n_{0}<n_{1}<\cdots<n_{d}$ with $n_{d}-n_{0} \leq m d$ and $\mu_{i_{n_{j}}}\left(A \cap \mathbb{S}_{n_{j}}\right) \geq 1 / m$ for $j=0,1, \ldots, d$. Let $\epsilon>0$. Concentration of measure on $\mathbb{S}_{n_{1}}$ implies that the measure of the $\epsilon / d$-neighbourhood of $A$ in $\mathbb{S}_{n_{1}}$ is $>1-C_{1} \exp \left(-C_{2} i_{n_{1}}\right)$. At the same time, Lemma 2.5 implies that the measure of the $\epsilon / d$-neighbourhood of $g_{1}\left(A \cap \mathbb{S}_{n}\right)$ in $\mathbb{S}_{n_{1}}$ is asymptotically (in $\left.n\right)$ greater than any function of the form $\exp \left(-C i_{n_{1}}\right)$. For $n$ sufficiently large, $\mathcal{O}_{\epsilon / d}(A) \cap \mathcal{O}_{\epsilon / d}\left(g_{1} A\right) \cap \mathbb{S}_{n_{1}}$ is a non-empty open subset of $\mathbb{S}_{i_{n_{1}}}$, and its measure is asymptotically $>>\exp \left(-C i_{n_{1}}\right)$ for every $C>0$. From here we deduce that $\mathcal{O}_{\epsilon / d}(A) \cap \mathcal{O}_{2 \epsilon / d}\left(g_{2} A\right) \cap \mathcal{O}_{2 \epsilon / d}\left(g_{2} g_{1} A\right) \cap \mathbb{S}_{n_{2}}$ is a non-empty open subset of $\mathbb{S}_{n_{2}}$ of positive measure $>>\exp \left(-C i_{n_{2}}\right)$. After $d$ inductive steps, we conclude that $\cap_{g \in F} \mathcal{O}_{\epsilon}(g A) \neq \emptyset$.

Remark 2.7. Is the above criterion true for locally compact groups and strongly continuous unitary representations? Even more generally (suggested by P. de la Harpe), the same can be asked about amenable unitary representations in the sense of Bekka [2]. For non locally compact topological groups the criterion fails if amenability is understood in the most natural sense of [12] (' $u$-amenability' of [7]), because $U(\mathcal{H})_{s}$ is $(u$-)amenable [7, 12], now cf. Corol. 2.3.

§3. Dynamical corollaries. Let $G$ be a topological group. The maximal $G$ compactification of a topological $G$-space $X$ is a compact $G$-space $\alpha_{G}(X)$ together with a morphism of $G$-spaces $i: X \rightarrow \alpha_{G}(X)$ such that any morphism from $X$ to a compact $G$-space factors through $i[18,9]$. By $G / H_{\gg}$ we denote the left factor-space $G / H$ of a topological group $G$ by a closed subgroup $H$, equipped with the uniformity whose basis is formed by entourages of the form $V_{\text {阝 }}=\left\{(x H, y H): x y^{-1} \in V\right\}$, where 
$V$ is a neighbourhood of $e_{G}$. The following is easily proved using standard tools of abstract topological dynamics [1, 9, 17, 18.

Proposition 3.1. The maximal $G$-compactification of the left topological G-space $G / H$ coincides with the Samuel compactification $\sigma\left(G / H_{\gg}\right)$.

Corollary 3.2. The pair $\left(G / H_{\gg}, G\right)$ has the concentration property if and only if $\alpha_{G}(G / H)$ has a fixed point.

The superscripts ' $u$ ' and ' $s$ ' will denote the uniform (respectively strong) operator topology on the unitary group. Since the sphere $\mathbb{S}_{\mathcal{H}}$ is both uniformly and as a $U(\mathcal{H})$-space isomorphic to $\left(U(\mathcal{H})_{u} / \mathrm{St}_{\xi}\right)_{\gg}$, where $\xi \in \mathbb{S}_{\mathcal{H}}$ is any, we obtain:

Corollary 3.3. The maximal $U(\mathcal{H})_{u}$-compactification of the unit sphere of a Hilbert space $\mathcal{H}$ has no fixed points.

(Cf. Stoyanov's theorem [15]: the maximal $U\left(l_{2}\right)_{s}$-compactification of $\mathbb{S}^{\infty}$ coincides with the unit ball of $l_{2}$ with the weak topology, and thus has a fixed point.)

A topological group $G$ is called extremely amenable (e.a.) [12, 13, 14] if every continuous action of $G$ on a compact space has a fixed point. It is equivalent to the existence of a fixed point in the greatest ambit $\mathcal{S}(G)$ [1, 18, 17, 14, that is, the Samuel compactification of $G_{\text {户 }}$.

Corollary 3.4. A topological group $G$ is extremely amenable if and only if the pair $\left(G_{\risingdotseq}, G\right)$ has the concentration property.

An example of an e.a. group is $U(\infty)_{u}$ [6].

Corollary 3.5. The Calkin group modulo constant multiples of the identity acts effectively on its universal minimal flow.

Remark 3.6. Contrary to what was in effect claimed in [6], concentration of measure on finite permutation groups [8] (cf. also [16]) does not lead to extreme amenability of the infinite symmetric group $S_{\infty}$, because the metric on the latter group as in [6], Remark 3.5, is not right invariant. In fact, $S_{\infty}$, equipped with the pointwise topology, admits continuous actions on compacta without fixed points [13]. This result, combined with a theorem of Gaughan [5], implies that there is no group topology making $S_{\infty}$ into a Lévy group [6, 4] even in a more general sense of [14].

Acknowledgements. I am grateful to Eli Glasner, whose paper [1] (as a 1996 preprint) and e-mail discussions have introduced me to the measure concentration phenomenon. Thanks to Pierre de la Harpe for very helpful remarks on the original version of this note. This investigation was partially supported by a 1997-2000 Marsden Fund grant for fundamental research VUW703.

\section{REFERENCES}

[1] J. Auslander, Minimal Flows and Their Extensions, North-Holland Mathematics Studies 153, North-Holland, Amsterdam-NY-London-Tokyo, 1988.

[2] M.E.B. Bekka, Amenable unitary representations of locally compact groups, Invent. Math. 100 (1990), 383-401. 
[3] R. Engelking, General Topology, PWN, Warczawa, 1977.

[4] S. Glasner, On minimal actions of Polish groups, Top. Appl. 85 (1998), 119-125.

[5] E.D. Gaughan, Topological group structures of infinite symmetric groups, Proc. Nat. Acad. Sci. USA 58 (1967), 907-910.

[6] M. Gromov and V.D. Milman, A topological application of the isoperimetric inequality, Amer. J. Math. 105 (1983), 843-854.

[7] P. de la Harpe, Moyennabilité de quelques groupes topologiques de dimension infinie, C.R. Acad. Sci. Paris, Sér. A 277 (1973), 1037-1040.

[8] B. Maurey, Constructions de suites symétriques, C.R. Acad. Sci. Paris, Sér. A-B 288 (1979), 679-681.

[9] M. Megrelishvili, Compactification and factorization in the category of G-spaces, in: Categorical Topology (J. Adamek and S. MacLane, eds.), Singapore, 1989, 220-237.

[10] V.D. Milman, Diameter of a minimal invariant subset of equivariant Lipschitz actions on compact subsets of $\mathbb{R}^{n}$, in: Geometrical Aspects of Functional Analysis (Israel Seminar, 1985-86), Lecture Notes in Math. 1267 (1987), Springer-Verlag, Berlin a.o., pp. 13-20.

[11] V.D. Milman, The heritage of P.Lévy in geometric functional analysis, Astérisque 157-158 (1988), 273-301.

[12] A.T. Paterson, Amenability, Math. Surveys and Monographs 29, Amer. Math. Soc., Providence, RI, 1988.

[13] V.G. Pestov, On free actions, minimal flows, and a problem by Ellis, Trans. Amer. Math. Soc. 350 (1998), 4149-4165.

[14] V. Pestov, Some universal constructions in abstract topological dynamics, in: Topological Dynamics and its Applications. A Volume in Honor of Robert Ellis, Contemp. Math. 215 (1998), 83-99.

[15] L. Stoyanov, Total minimality of the unitary groups, Math. Z. 187 (1984), 273-283.

[16] M. Talagrand, Concentration of measure and isoperimetric inequalities in product spaces, Publ. Math. IHES 81 (1995), 73-205.

[17] S. Teleman, Sur la représentation linéaire des groupes topologiques, Ann. Sci. Ecole Norm. Sup. 74 (1957), 319-339.

[18] J. de Vries, On the existence of G-compactifications, Bull. Acad. Polon. Sci. Math. Astron. Phys. 26 (1978), 275-280.

\section{§4. APPENDIX: technical details of some proofs in the Note}

\section{Proof of Proposition 1.1.}

(2) $\Rightarrow$ (3): emulates a proof of Proposition 4.1 and Theorem 4.2 in [10].

Firstly, there is a point $x^{*} \in \sigma X$ whose every neighbourhood is essential: assuming the contrary, one can cover $\sigma X$ with open $F$-inessential sets and select a finite subcover containing no $F$-essential sets, a contradiction.

Assume now $x^{*}$ is not a common fixed point for $F$, then for some $f \in F$ one has $f x^{*} \neq x^{*}$, and choosing an entourage, $W$, of the unique uniform structure on $\sigma X$ with $W^{2}\left[x^{*}\right] \cap W^{2}\left[f x^{*}\right]=\emptyset$, we conclude that $W\left[x^{*}\right]$ is $F$-inessential, a contradiction.

(3) $\Rightarrow$ (1): Without loss in generality and replacing $X$ with its separated reflection if necessary, one can assume that $X$ is a separated uniform space (that is, 
$\left.\cap \mathcal{U}=\Delta_{X}\right)$ : indeed, the Samuel compactifications of a uniform space $X$ and of its separated reflection are canonically homeomorphic.

If $\gamma$ is a finite cover of $X$, there is an $A$ with $\mathrm{cl}{ }_{\sigma X}(A)$ containing an $F$-fixed point, $x^{*}$. We claim that $A$ is essential.

To begin with, let us remind a simple fact of general topology: if $B, C \subseteq X$ are such that for some $V \in \mathcal{U}_{X}, V[B] \cap V[B]=\emptyset$, then $\operatorname{cl}_{\sigma X}(B) \cap \operatorname{cl}_{\sigma X}(C)=\emptyset$. To prove the fact, let $\rho$ be a uniformly continuous bounded pseudometric on $X$ with $(\rho(x, y)<1) \Rightarrow((x, y) \in V)$. Set $d_{B}(x)=\inf \{\rho(b, x): b \in B\}$; this function is uniformly continuous and bounded on $X$, and therefore extends to a continuous function $\tilde{d}$ on $\sigma X$. Since $d_{B}(b)=0$ for all $b \in B$ and $d_{B}(c) \geq 2$ for all $c \in C$, the same is true of the values of $\tilde{d}$ on elements of closure of $C$, and the statement follows.

Assume that $\cap_{f \in F_{1}} f(V[A])=\emptyset$ for some $V \in \mathcal{U}_{X}$, where $F_{1}$ is a finite subfamily of $F$. Since every $f \in F_{1}$ is a uniform isomorphism (and this is the only place in the proof where this assumption is actually used), it has a uniformly continuous inverse, $f^{-1}$, and there is an entourage $V_{1} \in \mathcal{U}_{X}$ with $(x, y) \in V_{1} \Rightarrow\left(f^{-1} x, f^{-1} y\right) \in V$ for all $x, y \in X$ and every $f \in F_{1}$. Equivalently, $(f x, f y) \in V_{1} \Rightarrow(x, y) \in V$. We conclude: $V_{1}[f(A)] \subseteq f(V[A])$ for each $f \in F_{1}$, and therefore $\cap_{f \in F_{1}} V_{1}[f(A)]=\emptyset$.

A finite induction in $|F|$, using the above stated fact from uniform topology shows that then $\cap_{f \in F} \mathrm{cl}_{\sigma X}(f(A))=\emptyset$. Since extensions of $f$ to $\sigma X$ are homeomorphisms, $\mathrm{cl}_{\sigma X}(f(A))=f\left(\mathrm{cl}_{\sigma X}(A)\right)$, and consequently $\cap_{f \in F_{1}} f\left(\mathrm{cl}_{\sigma X}(A)\right)=\emptyset$, a contradiction because the latter intersection contains $x^{*}$.

Proof of Proposition 2.1, implication (2) $\Rightarrow$ (1): Let $\phi$ be a left $G$-invariant mean on $C_{u}^{b}\left(\mathbb{S}_{2}\right)$, that is, a continuous positive linear functional of norm 1 , sending 1 to 1 and such that for every $f \in C_{u}^{b}\left(\mathbb{S}_{2}\right)$ and every $g \in G$ one has $\phi(f)=\phi(g f)$, where $(g f)(x)=f(g x), x, g \in G$. In other words, $\phi$ is a $G$-invariant state with respect to the left action of $G$ on the commutative $C^{*}$-algebra $C_{u}^{b}\left(\mathbb{S}_{2}\right)$.

For every Borel set $A \subseteq G$ and each $f \in \mathbb{S}_{2}$ set $z_{A}(f)=\left\|\chi_{A} \cdot f\right\|^{2}$, where $\chi_{A}$ denote, as usual, the characteristic function of $A$, and the dot stands for the muptiplication of (equivalence classes of) functions. Since the mapping $f \mapsto\|f\|^{2}$ is 2-Lipschitz on the unit sphere $\mathbb{S}_{2}$ of $L_{2}(G)$, so is the function $z_{A}: \mathbb{S}_{2} \rightarrow \mathbb{R}$. Being also bounded, $z_{A} \in C_{u}^{b}\left(\mathbb{S}_{2}\right)$. Set $m(A)=\phi\left(f_{A}\right)$. Each of the following properties is easy to verify.

1. $m(A) \geq 0$. (Indeed, for every Borel set $A$, the value $z_{A}(f) \geq 0$ for every function $f$, and $\phi$ is positive, that is, takes non-negative values at positive functions such as $z_{A}$.)

2. $m(A)=0$ for locally null sets $A$. (The function $\chi_{A}$ itself, and therefore the product $\chi_{A} \cdot f$, are equivalent to the null function.)

3. If $A \subseteq B$, then $m(A) \leq m(B)$. (In such a case $z_{A} \leq z_{B}$, and one again uses the positivity of $\phi$.)

4. $m(G)=1$. (Indeed, $z_{G} \equiv 1$ on the sphere $\mathbb{S}_{2}$, therefore $\phi\left(z_{G}\right)=1$.) 
5. If $A$ and $B$ are disjoint, then $m(A \cup B)=m(A)+m(B)$. (In this case, $z_{A \cup B}=z_{A}+z_{B}$, because for each $f \in \mathbb{S}_{2}, \chi_{A} f \perp \chi_{B} f$ and therefore

$$
\begin{aligned}
z_{A \cup B}(f) & =\left\|\chi_{A \cup B} f\right\|^{2}=\left\|\chi_{A} f+\chi_{B} f\right\|^{2} \\
& =\left\|\chi_{A} f\right\|^{2}+\left\|\chi_{B} f\right\|^{2}=z_{A}(f)+z_{B}(f),
\end{aligned}
$$

and one uses the linearity of $\phi$ to conclude that $m(A \cup B)=\phi\left(z_{A}+z_{B}\right)=$ $\left.\phi\left(z_{A}\right)+\phi\left(z_{B}\right)=m(A)+m(B).\right)$

6. The measure $m$ is left invariant, that if, for every Borel $A \subseteq G$ and $g \in G$, one has $m(g A)=m(A)$. (Indeed, $g z_{A}=z_{g^{-1} A}$, because

$$
\left(g z_{A}\right)(f)=z_{A}(g f)=\left\|\chi_{A} \cdot g f\right\|^{2}=\left\|\chi_{g^{-1} A} \cdot f\right\|^{2}=z_{g^{-1} A}(f),
$$

and the statement follows from left invariance of $\phi: m(g A)=\phi\left(z_{g A}\right)=$ $\phi\left(g^{-1} z_{A}\right)=\phi\left(z_{A}\right)=m(A)$.)

Thus, $m$ is a finitely-additive left-invariant normalized measure on Borel subsets of $G$, vanishing on locally null sets, and consequently $G$ is amenable.

Proof of Corollary 2.2. It is enough to make an obvious remark: every $U(\mathcal{H})$ invariant mean on $C_{u}^{b}\left(\mathbb{S}_{\mathcal{H}}\right)$ is invariant with respect to the action of every group $G$ represented in $\mathcal{H}$ by unitary operators. Since $\mathcal{H}$ is infinite-dimensional, one can find a non-amenable discrete group $G$ of the same cardinality as is the density character of $\mathcal{H}$, and to realize $\mathcal{H}$ as $l_{2}(G)$. (For example, take as $G$ the free group of rank equal to the density character of $\mathcal{H}$.) Now one can apply Proposition 2.1.

Proof of Corollary 2.3. If $\mathcal{H}$ is infinite-dimensional, the statement follows from Corollary 2.2. If $\operatorname{dim} \mathcal{H}<\infty$, the unitary group $U(n)$ possesses no fixed points in the compact sphere $\mathbb{S}_{\mathcal{H}}$, and there is no concentration property in a trivial way.

Proof of Lemma 2.5. Here is the statement of the Lemma again.

Let $\epsilon>0$. The measure of the $\epsilon$-neighbourhood of $\mathbb{S}^{n}$ in $\mathbb{S}^{n+k_{n}}$ is asymptotically greater than any function $\exp (-C n), C>0$, provided that $k_{n} / n \rightarrow 0$ as $n \rightarrow \infty$.

More precisely, whenever $k_{n}$ is a sequence of natural numbers with $\lim _{n \rightarrow \infty} k_{n} / n=$ 0 , then for every constant $C>0$

$$
\lim _{n \rightarrow \infty}\left[\frac{e^{-C n}}{\mu_{n+k_{n}}\left(\mathcal{O}_{\epsilon}\left(\mathbb{S}^{n}\right) \cap \mathbb{S}^{n+k_{n}}\right)}\right]=0 .
$$

Proof. By $\mu_{n}$ we will denote the (unique) normalized rotation-invariant Borel measure on the $n$-dimensional Euclidean sphere $\mathbb{S}^{n}$. The distances between points on the spheres will be geodesic distances.

Let $n \in \mathbb{N}$, and let $A$ be a subset of the sphere $\mathbb{S}^{n}$. By $S(A)$ we will denote the set of all points $x \in \mathbb{S}^{n+1}$ whose geodesic distance from the equatorial sphere $\mathbb{S}^{n} \subset \mathbb{S}^{n+1}$ is achieved at a point of $A$. If we denote by $\pi_{n}: \mathbb{R}^{n+1} \rightarrow \mathbb{R}^{n}$ the projection (deleting the last coordinate), then

$$
S(A)=\left\{x \in \mathbb{S}^{n+1}: \frac{\pi_{n}(x)}{\left\|\pi_{n}(x)\right\|} \in A\right\} \cup(0,0, \ldots, 0, \pm 1)
$$


Geometrically, $S(A)$ is the union of the family of all great circles passing through the north and south poles of $\mathbb{S}^{n+1}$ and containing an element of $A$. From the topological point of view, $S(A)$ is the suspension over $A$. The following is quite straightforward.

Lemma 4.1. $\mu_{n+1}(S(A))=\mu_{n}(A)$.

For an $\epsilon>0$, denote

$$
S_{\epsilon}(A)=S(A) \cap \mathcal{O}_{\epsilon}\left(\mathbb{S}^{n}\right)
$$

The set $S_{\epsilon}(A) \subseteq \mathbb{S}^{n+1}$ forms a 'spherical cylinder' with base $A$ and of geodesic height $\epsilon$ (provided $\epsilon<\pi / 2$, which is the case of interest). Transparent geometric considerations and Lemma 4.1 imply the following.

\section{Lemma 4.2.}

$$
\mu_{n+1}\left(S_{\epsilon}(A)\right)=\mu_{n+1}(S(A)) \cdot \mu_{n+1}\left(\mathcal{O}_{\epsilon}\left(\mathbb{S}^{n}\right) \cap \mathbb{S}^{n+1}\right) \equiv \mu_{n}(A) \cdot \mu_{n+1}\left(\mathcal{O}_{\epsilon}\left(\mathbb{S}^{n}\right) \cap \mathbb{S}^{n+1}\right)
$$

Now let $n<N$ be two natural numbers, and let $\epsilon>0$. Set $k=N-n$. Define a subset $\Omega \subseteq \mathbb{S}^{N}$ recursively as follows. Set $A_{0}=\mathbb{S}^{n}$. For every $j=1,2, \ldots, k$, set

$$
A_{j}=S_{\frac{\epsilon}{\sqrt{k}}}\left(A_{j-1}\right)
$$

Finally, set $\Omega=A_{k} \equiv A_{N-n} \subseteq \mathbb{S}^{N}$.

For every $j=0,1,2, \ldots, k$ denote $d_{j}=\sup \left\{d\left(x, \mathbb{S}^{n}\right): x \in A_{j}\right\}$. (For instance, $d_{0}=0$, while $d_{1}=\epsilon / \sqrt{k}$.)

Consider a point $x \in A_{j} \backslash A_{j-1} \subseteq \mathbb{S}^{n+j}$, 'newly-added' at a step $j=1,2, \ldots, k$. Let $x^{\prime}$ be the closest point to $x$ in $\mathbb{S}^{n+j-1}$ (and therefore in $A_{j-1}$ ). Let $x^{\prime \prime}$ be the closest point to $x^{\prime}$ in $\mathbb{S}^{n}$. The geodesic triangle in $\mathbb{S}^{n+1}$ with vertices at $x, x^{\prime}, x^{\prime \prime}$ is right-angled, with the length of the hypotenuse equal to the distance between $x$ and $x^{\prime \prime}$, and the two sides bounded above by $d_{j-1}$ and $\epsilon / \sqrt{k}$, respectively. Properties of spherical geometry imply that $d_{j}^{2}<d_{j-1}^{2}+\epsilon^{2} / k$. A finite induction in $j$ shows that $d_{k} \leq \epsilon$, that is, every point of $A_{k}$ is at a distance $<\epsilon$ from $A_{0}$, and

$$
\Omega \subseteq \mathcal{O}_{\epsilon}\left(\mathbb{S}^{n}\right) \cap \mathbb{S}^{N}
$$

Lévy's concentration of measure property for spheres (see e.g. [11]) implies the existence of constants $C_{1}, C_{2}>0$ (whose exact values will be of no importance here) with

$$
\mu_{n+1}\left(\mathcal{O}_{\epsilon}\left(\mathbb{S}^{n}\right) \cap \mathbb{S}^{n+1}\right) \geq 1-C_{1} \exp \left(-C_{2} \epsilon^{2}(n+1)\right)
$$


Using Lemma 4.2 recurrently, one gets the estimate:

$$
\begin{aligned}
\mu_{N}\left(\mathcal{O}_{\epsilon}\left(\mathbb{S}^{n}\right) \cap \mathbb{S}^{N}\right) \geq & \mu_{N}(\Omega) \\
\geq & \left(1-C_{1} \exp \left(-C_{2}\left(\frac{\epsilon}{\sqrt{k}}\right)^{2}(n+1)\right)\right) \\
& \times\left(1-C_{1} \exp \left(-C_{2}\left(\frac{\epsilon}{\sqrt{k}}\right)^{2}(n+2)\right)\right) \\
& \times \cdots \\
& \times\left(1-C_{1} \exp \left(-C_{2}\left(\frac{\epsilon}{\sqrt{k}}\right)^{2} N\right)\right) \\
\geq & \left(1-C_{1} \exp \left(-C_{2} \epsilon^{2} \frac{n}{k}\right)\right)^{k} \cdot
\end{aligned}
$$

Now let $C>0$ and $M>1$ be arbitrary. Since for $n / k$ large enough one has

$$
M \exp \left(-C \frac{n}{k}\right)+C_{1} \exp \left(-C_{2} \epsilon^{2} \frac{n}{k}\right)<1
$$

it follows that

$$
M^{k} \exp (-C n)<\left(1-C_{1} \exp \left(-C_{2} \epsilon^{2} \frac{n}{k}\right)\right)^{k}
$$

and finally

$$
\frac{\exp (-C n)}{\mu_{N}\left(\mathcal{O}_{\epsilon}\left(\mathbb{S}^{n}\right) \cap \mathbb{S}^{N}\right)}<\frac{\exp (-C n)}{\left(1-C_{1} \exp \left(-C_{2} \epsilon^{2} \frac{n}{k}\right)\right)^{k}}<\frac{1}{M^{k}} \leq \frac{1}{M},
$$

provided $n / k$ is large enough, that is, whenever $k / n$ is sufficiently close to zero. This finishes the proof of Lemma 2.5.

Proof of Theorem 2.6. Since $\Leftarrow$ follows from Prop. 2.1, only $\Rightarrow$ needs proving.

First of all, we will establish an auxiliary Lemma.

Lemma 4.3. Let $n, N \in \mathbb{N}$ and $\epsilon>0$ be arbitrary, where $n \leq N$ and $\epsilon<\pi / 2$. Let $A$ be a Borel subset of the sphere $\mathbb{S}^{n}$. Then

$$
\mu_{N}\left(\mathcal{O}_{\epsilon}(A) \cap \mathbb{S}^{N}\right) \geq \mu_{n}(A) \cdot \mu_{N}\left(\mathcal{O}_{\epsilon}\left(\mathbb{S}^{n}\right) \cap \mathbb{S}^{N}\right)
$$

Remark 4.4. The above Lemma says that the proportion of the the $\epsilon$-neighbourhood of $\mathbb{S}^{n}$ in a sphere of larger dimension $\mathbb{S}^{N}$, taken up by the $\epsilon$-neighbourhood of $A$, is at least as large as the proportion of $A$ in $\mathbb{S}^{n}$.

Proof. The $\epsilon$-neighbourhood of $\mathbb{S}^{n}$ in $\mathbb{S}^{N}$ forms a fibre bundle over $\mathbb{S}^{n}$ in a canonical sort of way: if $\mathbb{S}^{n}$ is identified with the set of all elements $x$ of the unit sphere in $\mathbb{R}^{N}$ whose $i$-th coordinates vanish, $i=n+1, \ldots, N$, then the projection mapping of the fibre bundle, $p: \mathcal{O}_{\epsilon}\left(\mathbb{S}^{n}\right) \cap \mathbb{S}^{N} \rightarrow \mathbb{S}^{n}$, takes an element $x=\left(x_{1}, \ldots, x_{N}\right)$ to $\left\|\left(x_{1}, \ldots, x_{n}\right)\right\|^{-1} \cdot\left(x_{1}, \ldots, x_{n}\right)$. Denote by $\tilde{A}=p^{-1}(A)$ the complete preimage of $A$ 
in $\mathcal{O}_{\epsilon}\left(\mathbb{S}^{n}\right) \cap \mathbb{S}^{N}$ under the projection mapping. A simple calculation shows that $\tilde{A}$ is contained in the $\epsilon$-neighbourhood of $A$, and one has

$$
\frac{\int_{\mathcal{O}_{\epsilon}(A) \cap \mathbb{S}^{N}} d \mu_{N}}{\int_{\mathcal{O}_{\epsilon}\left(\mathbb{S}^{n}\right) \cap \mathbb{S}^{N}} d \mu_{N}} \geq \frac{\int_{\tilde{A}} d \mu_{N}}{\int_{\mathcal{O}_{\epsilon}\left(\mathbb{S}^{n}\right) \cap \mathbb{S}^{N}} d \mu_{N}}=\frac{\int_{A} d \mu_{n}}{\int_{\mathbb{S}^{n}} d \mu_{n}},
$$

and the statement follows.

Let $F \subseteq G$ be an arbitrary finite set, $d=|F|$. Choose a subset $D=\left\{g_{1}, g_{2}, \ldots, g_{d}\right\} \subseteq$ $G$ such that the products of the form $g_{d}, g_{d} \cdot g_{d-1}, \ldots, g_{d} \cdot g_{d-1} \cdots g_{2}$, $g_{d} \cdot g_{d-1} \cdots \cdot g_{2} \cdot g_{1}$ exhaust all of $F$.

Using the Følner condition, choose for every $n \in \mathbb{N}$ a finite set $K_{n} \subseteq G$ with the property

$$
\frac{\left|K_{n} \Delta\left(D \cdot K_{n}\right)\right|}{\left|K_{n}\right|} \rightarrow 0
$$

as $n \rightarrow \infty$. Moreover, assume that for every $n \in \mathbb{N}$,

$$
D \cdot K_{n} \subseteq K_{n+1}
$$

(E.g. by using the Følner condition in the form of [12], Th. 4.13,(iii).)

Choose an increasing chain of vector spaces $V_{n} \subset \mathcal{H}$ of finite dimension $i_{n}=$ $\operatorname{dim} V_{n} \geq n$ such that

$$
\pi_{g} \cdot V_{n} \subseteq V_{n+1} \text { for all } g \in D
$$

and

$$
\frac{i_{n+1}}{i_{n}} \equiv \frac{\operatorname{dim} V_{n+1}}{\operatorname{dim} V_{n}} \leq \frac{\left|K_{n} \cup\left(D \cdot K_{n}\right)\right|}{\left|K_{n}\right|} \rightarrow 0 .
$$

Such a choice is possible. Indeed, if $\pi$ admits a cyclic $G$-submodule of $\mathcal{H}$ having infinite dimension, that is, there is a vector $\xi \in \mathcal{H}$ whose $G$-orbit under the representation $\pi$ is infinite, then one simply takes as $V_{n}$ the linear span of $\left\{\pi_{g} \xi: g \in K_{n}\right\}$ in $\mathcal{H}$. If such a $\xi$ does not exist, then the situation becomes in a sense trivial: $\mathcal{H}$ decomposes into an infinite direct $l_{2}$-sum of $G$-submodules of dimension $\leq N$, where $N$ is a fixed natural number, $\mathcal{H}=\bigoplus_{i \in I} \mathcal{H}_{i}$. Now it is enough to choose a countably infinite set $\left\{i_{j}: j \in \mathbb{N}\right\} \subseteq I$ and set $V_{n}=\oplus_{j=1}^{n} \mathcal{H}_{i_{j}}$. (In this case, $i_{n+1} \leq i_{n}+N$.)

Denote $\mathbb{S}_{n}=\mathbb{S} \cap V_{n}$ and $k_{n}=i_{n+1}-i_{n}$. One has by the choice of $V_{n}$ :

$$
\frac{k_{n}}{i_{n}} \rightarrow 0 \text { as } n \rightarrow \infty \text {. }
$$

Let now $\gamma$ be an arbitrary finite cover of $\mathbb{S}$, and denote $m=|\gamma|$.

Since $d$ and $m$ are constants fixed for the rest of the proof, one has

$$
\lim _{n \rightarrow \infty} \frac{i_{n+d m}}{i_{n}}=\lim _{n \rightarrow \infty} \frac{i_{n+d m}}{i_{n+d m-1}} \lim _{n \rightarrow \infty} \frac{i_{n+d m-1}}{i_{n+d m-2}} \cdots \lim _{n \rightarrow \infty} \frac{i_{n+1}}{i_{n}}=1^{d m}=1 .
$$

Let $\mu_{n}$, as before, stand for the rotation-invariant probability measure on the $n$-dimensional unit Euclidean sphere $\mathbb{S}^{n}$. For every $A \in \gamma$, set

$$
A^{\sim}=\left\{n \in \mathbb{N}: \mu_{i_{n}}\left(A \cap \mathbb{S}_{n}\right)\right\} \geq 1 / m
$$


The collection $\left\{A^{\sim}: A \in \gamma\right\}$ forms a finite cover of the set of natural numbers $\mathbb{N}$. It follows that for at least one $A \in \gamma$, the natural numbers $n$ with the property

$$
\left|A^{\sim} \cap[n, n+d m]\right| \geq d
$$

form an infinite set. (Assuming the contrary implies that all sufficiently large natural numbers $n \in \mathbb{N}$ have the property that for every $A \in \gamma,\left|A^{\sim} \cap[n, n+d m]\right|<d$ and consequently $|[n, n+d m]|<d m$.) Fix such an $A \in \gamma$ once and for all.

We are going to prove now that the set $A \in \gamma$ so chosen is $F$-essential. Let $\epsilon>0$ be arbitrary. We will show that

$$
\cap_{g \in F} \mathcal{O}_{\epsilon}(A) \neq \emptyset
$$

thus finishing the proof of the Theorem. The latter condition can be rewriteen as

$$
\mathcal{O}_{\epsilon}(A) \cap g_{1} \mathcal{O}_{\epsilon}(A) \cap g_{2} g_{1} \mathcal{O}_{\epsilon}(A) \cap \cdots \cap g_{d} g_{d-1} \cdots g_{2} g_{1} \mathcal{O}_{\epsilon}(A) \neq \emptyset,
$$

or else (because the representation is unitary)

$$
\mathcal{O}_{\epsilon}(A) \cap \mathcal{O}_{\epsilon}\left(g_{1} A\right) \cap \mathcal{O}_{\epsilon}\left(g_{2} g_{1} A\right) \cap \cdots \cap \mathcal{O}_{\epsilon}\left(g_{d} g_{d-1} \cdots g_{2} g_{1} A\right) \neq \emptyset .
$$

For every $n \in \mathbb{N}$ fix, using (23), a collection of $d+1$ natural numbers

$$
n \leq n_{0}<n_{1} \leq n_{2}<\cdots<n_{d}
$$

with the property that $n_{d}-n_{0} \leq m d$ and $n_{0}, n_{1}, n_{2}, \ldots, n_{d} \in A^{\sim}$. (Notice that all $n_{i}$ are functions of $n$ rather than constant values, because we are interested in the exponential behaviour as $n \rightarrow \infty$ of all the quantities involved.)

According to the Lévy concentration property applied to the spheres $\mathbb{S}_{n_{1}}$, the $i_{n_{1}}$ measure of the $\epsilon / d$-neighbourhood of $A$ in $\mathbb{S}_{n_{1}}$ approaches 1 exponentially fast as $n \rightarrow \infty$. A more precise statement is that such a measure is $\geq 1-C_{1} \exp \left(-C_{2} i_{n_{1}}\right) \geq$ $1-C_{1} \exp \left(-C_{2} i_{n_{0}}\right)$.

At the same time, Lemmas 4.3 and 2.5 tell us that the measure of the $\epsilon / d$ neighbourhood of $g_{1}\left(A \cap \mathbb{S}_{n_{0}}\right)$ in $\mathbb{S}_{n_{1}}$ is greater than or equal to

$$
\mu_{i_{n}}\left(A \cap \mathbb{S}_{n_{0}}\right) \cdot \mu_{i_{n_{1}}}\left(\mathcal{O}_{\epsilon / d}\left(\mathbb{S}_{n_{0}}\right) \cap \mathbb{S}_{n_{1}}\right) \geq \frac{1}{2} \mu_{i_{n_{1}}}\left(\mathcal{O}_{\epsilon / d}\left(\mathbb{S}_{n_{0}}\right) \cap \mathbb{S}_{n_{1}}\right)
$$

and since $\left(i_{n_{1}}-i_{n_{0}}\right) / i_{n_{0}} \leq\left(i_{n+m d}-i_{n_{0}}\right) / i_{n_{0}} \leq k_{n} / i_{n} \rightarrow 0$ as $n \rightarrow \infty$, the number on the r.h.s. in (28) is asymptotially (in $n$ ) greater than any function of the form $\exp \left(-C i_{n_{0}}\right)$. In particular, for $n$ sufficiently large

$$
\mu_{i_{n_{1}}}\left(\mathcal{O}_{\epsilon / d}\left(g_{1}\left(A \cap \mathbb{S}_{n_{0}}\right)\right) \cap \mathbb{S}_{n_{1}}\right)>1-\mu_{i_{n_{1}}}\left(\mathcal{O}_{\epsilon / d}(A) \cap \mathbb{S}_{n_{1}}\right)
$$

so that the $\epsilon / d$-neighbourhoods of $A$ and of $g_{1} A$ have a non-empty common intersection with $\mathbb{S}_{n_{1}}$. Denote

$$
V_{1}=\mathbb{S}_{n_{1}} \cap \mathcal{O}_{\epsilon / d}(A) \cap \mathcal{O}_{\epsilon / d}\left(g_{1} A\right)
$$

Since $V_{1}$ is non-empty and open in $\mathbb{S}_{n_{1}}$, it is of positive measure. To stress that the value of the measure is a function of $n$, we will denote it by $m_{1}(n)=\mu_{i_{n_{1}}}\left(V_{1}\right)$. It might well be the case that as $n \rightarrow \infty$, one has $m_{1}(n) \rightarrow 0$. Nevertheless, it follows from Lemma 2.5 that asymptotically

$$
m_{1}(n)>\exp \left(-C i_{n_{0}}\right) \geq \exp \left(-C i_{n_{1}}\right) \text { for every } C>0 .
$$


Now let us perform a step of induction. Let $j=1, \ldots, k$, and suppose we have proved that the open subset

$$
\begin{aligned}
V_{j}= & \mathbb{S}_{n_{j}} \cap \mathcal{O}_{\epsilon / d}(A) \cap \mathcal{O}_{2 \epsilon / d}\left(g_{j} A\right) \cap \mathcal{O}_{3 \epsilon / d}\left(g_{j} g_{j-1} A\right) \cap \cdots \\
& \cap \mathcal{O}_{(d-j) \epsilon / d}\left(g_{j} g_{j-1} \cdots g_{2} A\right) \cap \mathcal{O}_{(d-j) \epsilon / d}\left(g_{j} g_{j-1} \cdots g_{2} g_{1} A\right)
\end{aligned}
$$

of the sphere $\mathbb{S}_{n_{j}}$ is non-empty for all $n$ large enough, and moreover the measure of this set has the property

$$
m_{j}(n) \equiv \mu_{i_{n_{j}}}\left(V_{j}\right)>e^{-C i_{n_{j}}} \text { asymptotically as } n \rightarrow \infty \text {, for every } C>0 \text {. }
$$

The set $g_{j+1} V_{j}$ is contained in the $i_{n_{j}}$-dimensional sphere $g_{j+1}\left(\mathbb{S}_{n_{j}}\right) \subset \mathbb{S}_{n_{j+1}}$. It follows from Lemma 4.3 and Lemma 2.5 that

$$
\begin{aligned}
\mu_{i_{n_{j+1}}}\left(\mathcal{O}_{\epsilon / d}\left(g_{j+1}\left(V_{j}\right)\right) \cap \mathbb{S}_{n_{j+1}}\right) & \geq \mu_{i_{n_{j}}}\left(V_{j}\right) \cdot \mu_{i_{n_{j+1}}}\left(\mathcal{O}_{\epsilon / d}\left(\mathbb{S}_{n_{j}}\right) \cap \mathbb{S}_{n_{j+1}}\right) \\
& >\exp \left(-C^{\prime} i_{n_{j}}\right) \exp \left(-C^{\prime \prime} i_{n_{j}}\right) \\
& \geq \exp \left(-C i_{n_{j}}\right)
\end{aligned}
$$

asymptotically in $n$ for every $C>0$, because $\left(i_{n_{j+1}}-i_{n_{j}}\right) / i_{n_{j}}<k_{n} / i_{n} \rightarrow 0$.

At the same time, the $i_{n_{j+1}}$-measure of $\mathcal{O}_{\epsilon / d}(A) \cap \mathbb{S}_{n_{j+1}}$ approaches 1 exponentially fast as $n \rightarrow \infty$, or, more accurately, is greater than $1-C_{1} \exp \left(-C_{2} i_{n_{j+1}}\right) \geq 1-$ $C_{1} \exp \left(-C_{2} i_{n_{j}}\right)$. It follows that, for $n$ large enough,

$$
\mu_{i_{n_{j+1}}}\left(\mathcal{O}_{\epsilon / d}\left(g_{j+1}\left(V_{j}\right)\right) \cap \mathbb{S}_{n_{j+1}}\right)>1-\mu_{i_{n_{j+1}}}\left(\mathcal{O}_{\epsilon / d}(A) \cap \mathbb{S}_{n_{j+1}}\right)
$$

so that the $\epsilon / d$-neighbourhoods of $A$ and $g_{j+1} V_{j}$ have a non-empty common intersection with $\mathbb{S}_{n_{j+1}}$ :

$$
\mathbb{S}_{n_{j+1}} \cap \mathcal{O}_{\epsilon / d}(A) \cap \mathcal{O}_{\epsilon / d}\left(g_{j+1} V_{j}\right) \neq \emptyset
$$

Notice that

$$
\begin{aligned}
g_{j+1} V_{j}= & g_{j+1} \mathbb{S}_{n_{j}} \cap \mathcal{O}_{\epsilon / d}\left(g_{j+1} A\right) \cap \mathcal{O}_{2 \epsilon / d}\left(g_{j+1} g_{j} A\right) \cap \mathcal{O}_{3 \epsilon / d}\left(g_{j+1} g_{j} g_{j-1} A\right) \cap \cdots \\
& \cap \mathcal{O}_{(d-j) \epsilon / d}\left(g_{j+1} g_{j} g_{j-1} \cdots g_{2} A\right) \cap \mathcal{O}_{(d-j) \epsilon / d}\left(g_{j+1} g_{j} g_{j-1} \cdots g_{2} g_{1} A\right) .
\end{aligned}
$$

Since the $\delta$-neighbourhood of the intersection of sets is contained in the intersection of $\delta$-neighbourhoods of the sets, one has

$$
\begin{aligned}
\mathcal{O}_{\epsilon / d}\left(g_{j+1} V_{j}\right) \subseteq & \mathcal{O}_{2 \epsilon / d}\left(g_{j+1} A\right) \cap \mathcal{O}_{3 \epsilon / d}\left(g_{j+1} g_{j} A\right) \cap \mathcal{O}_{4 \epsilon / d}\left(g_{j+1} g_{j} g_{j-1} A\right) \cap \cdots \\
& \cap \mathcal{O}_{(d-j+1) \epsilon / d}\left(g_{j+1} g_{j} g_{j-1} \cdots g_{2} A\right) \\
& \cap \mathcal{O}_{(d-j+1) \epsilon / d}\left(g_{j+1} g_{j} g_{j-1} \cdots g_{2} g_{1} A\right)
\end{aligned}
$$

so that

$$
\begin{aligned}
V_{j+1}:= & \mathbb{S}_{n_{j+2}} \cap \mathcal{O}_{\epsilon / d}(A) \cap \mathcal{O}_{2 \epsilon / d}\left(g_{j+1} A\right) \cap \mathcal{O}_{3 \epsilon / d}\left(g_{j+1} g_{j} A\right) \cap \cdots \\
& \cap \mathcal{O}_{(d-j+1) \epsilon / d}\left(g_{j+1} g_{j} g_{j-1} \cdots g_{2} A\right) \\
& \cap \mathcal{O}_{(d-j+1) \epsilon / d}\left(g_{j+1} g_{j} g_{j-1} \cdots g_{2} g_{1} A\right)
\end{aligned}
$$

is a non-empty open subset of $\mathbb{S}_{n_{j+1}}$ of positive measure $m_{j+1}(n)$, which asymptotically in $n$ behaves as

$$
m_{j+1}(n)>>\exp \left(-C i_{n_{j}}\right) \geq \exp \left(-C i_{n_{j+1}}\right) \text { for every } C>0 .
$$

A step of induction has been performed. 
At the last step $j=d$, we conclude that for $n$ sufficiently large,

$$
V_{d}:=\mathbb{S}_{n_{d}} \cap \mathcal{O}_{\epsilon / d}(A) \cap \mathcal{O}_{2 \epsilon / d}\left(g_{d} A\right) \cap \mathcal{O}_{3 \epsilon / d}\left(g_{d} g_{d-1} A\right) \cap \cdots \cap \mathcal{O}_{\epsilon}\left(g_{d} g_{d-1} \cdots g_{2} g_{1} A\right)
$$

is an open non-empty subset of $\mathbb{S}_{n_{d}}$, and thus (26) is established.

The statement of the Theorem follows now by force of Proposition 4.5 below.

Proposition 4.5. Let $X=\left(X, \mathcal{U}_{X}\right)$ be a uniform space, and let $G$ be a family of uniform self-mappings of $X$. The pair $(X, F)$ has the property of concentration if and only if for every finite collection $f_{1}, f_{2}, \ldots, f_{n} \in G, n \in \mathbb{N}$, and every finite cover $\gamma$ of $X$ there is an F-essential $A \in \gamma$. (That is, $A$ is such that for every $V \in \mathcal{U}_{X}$ one has

$$
\left.\cap_{i=1}^{n} f_{i} V[A] \neq \emptyset .\right)
$$

Remark 4.6. The difference with the original definition of the concentration property is that instead of requesting every finite cover $\gamma$ of $X$ to contain a subset that is $F$-essential for every finite set of transformations $F$, we request $\gamma$ to contain an $F$-essntial set for each finite $F$.

Proof. Of course, the difference between two formulations is only superficial. Only $\Leftarrow$ needs proof. Denote for every finite $F$ by $\gamma_{F}$ the collection of all $F$-essential elements of $\gamma$. Clearly, whenever $F_{1} \subseteq F_{2}$, one must have $\gamma_{F_{2}} \subseteq \gamma_{F_{1}}$. The compactness (or rather finiteness) considerations lead one to conclude that

$$
\bigcap_{F \subseteq G,|F|<\infty} \gamma_{F} \neq \emptyset
$$

thus finishing the proof: every element $A$ of the above intersection is $G$-essential.

Proof of the Proposition 3.1. The argument outlined below is really a commonplace in abstract topological dynamics, and surely the statement of Proposition must have been stated somewhere; it is just that I was unable to find a reference.

The Samuel compactification of $G / H_{\gg}$ is the compactification determined by all $\mathcal{U}_{\Gamma}$-uniformly continuous $\left(\mathcal{U}_{\Gamma}\right.$-u.c.) functions $f: G / H \rightarrow \mathbb{R}$, that is, functions satisfying the condition: for every $\epsilon>0$, there is a $V \ni e$ such that

$$
\forall x, y \in G, x y^{-1} \in V \Rightarrow|f(x H)-f(y H)|<\epsilon
$$

(We deliberately avoid using the 'right/left uniformly continuous' terminology, because the mathematical community seems to be divided into two groups of roughly the same size, one of them calling the $\mathcal{U}_{\vec{r}}$-u.c. functions 'right' uniformly continuous, the other 'left' uniformly continuous. Our system of notation, suggested in [13, 14, has the mnemonic advantage of the symbol $\ulcorner$ graphically indicating the positioning of the inverse symbol in the expression $x y^{-1}$. The functions satisfying the property

$$
\forall x, y \in G, x^{-1} y \in V \Rightarrow|f(x H)-f(y H)|<\epsilon
$$

are called by us $\mathcal{U}_{\uparrow-\text {-uniformly continuous.) }}$ 


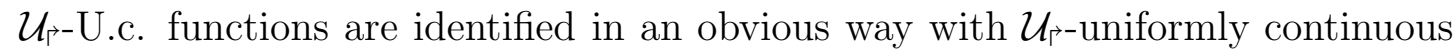
functions on $G$ that are constant on all left cosets $x H, x \in G$. Their totality forms a $G$-invariant $C^{*}$-subalgebra, which we will denote $C_{u}^{b}\left(G / H_{\risingdotseq}\right)$, of $C_{u}^{b}\left(G_{\risingdotseq}\right)$. Since the left regular representation of $G$ in $C_{u}^{b}\left(G_{\risingdotseq}\right)$ (defined by $(g f)(x)=f(g x)$ ) is well known (and easily checked) to be strongly continuous [17, 18, 1], 14], so is the subrepresentation of $G$ in $C_{u}^{b}\left(G / H_{队}\right)$. Now it follows from a result of Teleman 177 that the action of $G$ on the Gelfand spectrum of $C_{u}^{b}\left(G / H_{\rightleftarrows}\right)$ is continuous, that is, $\sigma\left(G / H_{\text {队 }}\right)$ is a topological $G$-space. The uniformly continuous mapping of compactification $G / H_{\gg} \rightarrow \sigma\left(G / H_{\gg}\right)$ has the everywhere dense image and is readily checked to be $G$-equivariant.

It only remains to prove the maximality of $\sigma\left(G / H_{\risingdotseq}\right)$. This is done in a standard fashion. Let $X$ be a compact $G$-space, and let $\phi: G / H \rightarrow X$ be a continuous $G$ equivariant mapping. It determines a morphism, $\phi^{*}$, of $C^{*}$-algebras from $C(X)$ to $C_{u}^{b}\left(G / H_{\risingdotseq}\right)$ via

$$
C(X) \ni f \mapsto[(x H) \mapsto \tilde{f}(x H):=f(\phi(x))] \in C_{u}^{b}\left(G / H_{\longmapsto}\right)
$$

The dual continuous mapping $f^{\sim}: \sigma\left(G / H_{\text {户 }}\right) \rightarrow X$ between the corresponding Gelfand spaces is $G$-equivariant and its restriction to $G / H$ is easily seen to coincide with $f$. The proof is thus finished.

Proof of Corollary 3.5. Recall that the Calkin group is the topological factorgroup of $U\left(l_{2}\right)_{u}$ by the closure of $U(\infty)$ (in the uniform topology). This closure, $\overline{U(\infty)}$, is a normal subgroup of $U\left(l_{2}\right)$, consisting of all operators of the form $\mathbb{I}+T$, where $T$ is compact.

Proposition 3.1 tells us that the action of $U(\mathcal{H})_{u}$ upon $\sigma\left(\mathbb{S}_{\mathcal{H}}\right)$ is continuous for every Hilbert space $\mathcal{H}$, that is, $\sigma\left(\mathbb{S}_{\mathcal{H}}\right)$ forms a $U(\mathcal{H})_{u}$-flow (or a compact $U(\mathcal{H})_{u^{-}}$ space).

Denote by $\mathbb{T}$ the subgroup of $U\left(l_{2}\right)$ consisting of all scalar multiples of the identity $\lambda \mathbb{I}$, where $\lambda \in \mathbb{C}$ and $|\lambda|=1$. Let us recall the result by Gromov and Milman ([6], Example 5.1): if a compact group $G$ acts by isometries on the unit sphere $\mathbb{S}^{\infty}$ of $l_{2}$, then the pair $\left(\mathbb{S}^{\infty}, G\right)$ has the concentration property. It means that there exists a $\mathbb{T}$-fixed point $x_{1} \in \sigma\left(\mathbb{S}^{\infty}\right)$. Denote by $\mathfrak{X}$ the closure of the $U\left(l_{2}\right)$-orbit of $x_{1}$ in $\sigma\left(\mathbb{S}^{\infty}\right)$. It is a compact $U\left(l_{2}\right)_{u}$-space of $\sigma\left(\mathbb{S}^{\infty}\right)$. Since $\mathbb{T}$ is the centre of $U\left(l_{2}\right)$, every point of $\mathfrak{X}$ is $\mathbb{T}$-fixed. (In particular, it follows that $\mathfrak{X}$ is always a proper subspace of $\sigma\left(\mathbb{S}^{\infty}\right)$.)

Every compact $G$-flow contains a minimal subflow (that is, a compact $G$-subspace such that the orbit of each point is everywhere dense in it, see e.g. [1]), and since $U\left(l_{2}\right)$ has no fixed points in $\sigma\left(\mathbb{S}^{\infty}\right)$, it follows that every minimal $U\left(l_{2}\right)$-subflow of $\sigma\left(\mathbb{S}^{\infty}\right)$ is nontrivial, that is, contains more than one point. In particular, $\mathfrak{X}$ contains a minimal $U\left(l_{2}\right)$-subflow, which we will denote by $\mathcal{M}$, and which is nontrivial. Notice again that the action of $\mathbb{T}$ leaves each element of $\mathcal{M}$ fixed.

We need another result by Gromov and Milman [6]: the group $U(\infty)$ is extremely amenable, that is, has a fixed point in each compactum it acts upon continuously. Such a point therefore exists in $\mathcal{M}$. Denote this fixed point by $x^{*}$. It follows from the continuity of the action that $x^{*}$ is a fixed point for $\overline{U(\infty)}$, that is, the stabilizer 
$\mathrm{St}_{x^{*}}$ contains $\overline{U(\infty)}$. Since every point of $\mathcal{M}$ is fixed under $\mathbb{T}$ as well, it follows that $x^{*}$ is fixed under the action of the group $\mathbb{T} \cdot \overline{U(\infty)}$.

Now, it follows from general facts of topological dynamics that the stabilizers of elements of the orbit of $x^{*}$ under the action of $U\left(l_{2}\right)$ are conjugate to $\mathrm{St}_{x^{*}}$. Since $\mathbb{T} \cdot \overline{U(\infty)}$ is normal in $U\left(l_{2}\right)$, every such stabilizer contains $\mathbb{T} \cdot \overline{U(\infty)}$. Because of minimality of $\mathcal{M}$, the $U\left(l_{2}\right)$-orbit of $x^{*}$ is everywhere dense in $\mathcal{M}$, and we conclude: all points of $\mathcal{M}$ are fixed under the action of $\mathbb{T} \cdot \overline{U(\infty)}$. It implies that the action of $U\left(l_{2}\right)_{u}$ on $\mathcal{M}$ factors through an action of the projective Calkin group $U\left(l_{2}\right)_{u} /(\mathbb{T} \cdot \overline{U(\infty)})$, and the latter action is continuous.

The group $\mathbb{T} \cdot \overline{U(\infty)}$ forms the largest proper closed normal subgroup of $U\left(l_{2}\right)_{u}$. This was proved by Kadisonf and, as was pointed to me by P. de la Harpe, the statement remains true even without the word 'closed.' 9

Denote by $K$ the set of all $u \in U\left(l_{2}\right)_{u}$ leaving each element of $\mathcal{M}$ fixed. This is a closed normal subgroup of $U\left(l_{2}\right)_{u}$, containing $\overline{U(\infty)}$, and since it is proper, it must be contained in $\mathbb{T} \cdot \overline{U(\infty)}$ and consequently coincide with it. It means that the action of the projective Calkin group is effective, and the statement is proved.

Elaborating on the Remark 3.6. Gaughan's result [5] states that every Hausdorff group topology on the infinite symmetric group $S(X)$ contains the topology of pointwise convergence on $X$ (that is, the topology induced from the Tychonoff power, $X^{X}$, of the space $X$ equipped with the discrete topology). Denote by $S_{p}(X)$ the symmetric group equipped with the pointwise topology; it is well-known to form a Hausdorff topological group. If $\tau$ is a Hausdorff group topology on $S(X)$, then the identity isomorphism $(S(X), \tau) \rightarrow S_{p}(X)$ is always continuous. According to a recent result by the present author [13], $S_{p}(X)$ admits fixed point free actions on compacta. By composing this action with the continuous group isomorphism $(S(X), \tau) \rightarrow S_{p}(X)$, one deduces the following result.

Corollary 4.7. The group $S(X)$ equipped with an arbitrary Hausdorff group topology admits a fixed point free action on a compact space. In particular, it is not a Lévy group.

Here the concept of a Lévy group [6, 14] can be understood in the more general sense of [14], where metrizability is not presumed and the uniform structure is used in the definition instead. It implies that the concentration of measure cannot be observed on the family of finite symmetric groups $S(n)$ with respect to a rightinvariant metric generating a group topology on $S_{\infty}$.

The Hamming distance is given on each group of permutations $S(n)$ by

$$
d\left(\sigma_{1}, \sigma_{2}\right)=\left|\left\{i: \sigma_{1}(i) \neq \sigma_{2}(i)\right\}\right| .
$$

While the group $S_{\infty}^{f}$ of all finite permutations of a countably infinite set (e.g. $\mathbb{N}$ ) can be represented as the union of an increasing chain of finite permutation groups $S_{n}$,

\footnotetext{
${ }^{1}$ R. Kadison, Infnite unitary groups, Trans. Amer. Math. Soc. 72 (1952), 386-399.

${ }^{2} \mathrm{P}$. de la Harpe, Simplicity of the projective unitary groups defined by simple factors, Comment. Math. Helv. 54 (1979), 334-345.
} 
Corollary 4.7 says that there is no 'coherent' way of putting together the normalised Hamming distances so as to obtain a right-invariant metric on $S_{\infty}^{f}$.

Let us analyse one concrete example. In [6], Remark 3.5, it was suggested to define a function

$$
\varphi(\sigma, \eta)= \begin{cases}\frac{d(\sigma, \eta)}{\max \{d(\sigma, e), d(\eta, e)\}}, & \text { if } \sigma \neq \eta, \\ 0 & \text { otherwise }\end{cases}
$$

and then to find a metric, $\hat{d}$, on $S_{\infty}$, determining the topology of the latter group and Lipschitz equivalent to $\varphi$ with Lipschitz constant 2.

Such a metric of course does exist. However, it must be clear from the results of the present paper that what really matters for concentration property and the existence of fixed points in compactifications, is not the topology of a topological group $G$ per se, but the uniform structure $\mathcal{U}_{\gg}$ of $G$. Let us show that the uniform structure generated by $\hat{d}$ has the property that the right translations of $S_{\infty}$ do not form a right equicontinuous family, and therefore this uniform structure does not coincide with the uniform structure $\mathcal{U}_{\triangleright}$ of any group topology on $S_{\infty}$ (in particular, that of the natural - pointwise - topology on $\left.S_{\infty}\right)$. (Notice that if $(x, y) \in V_{\text {阝 }}$ and $g \in G$, then $(x g)(y g)^{-1}=x g g^{-1} y^{-1}=x y^{-1} \in V$, that is, $(x g, y g) \in V_{\text {阝 as well, }}$ hence the equicontinuity property for right translations follows.)

In view of the Lipschitz equivalence of $\hat{d}$ and $\varphi$, the uniformity generated by $\hat{d}$ coincides with that generated on $S_{\infty}$ by $\varphi$ through taking as the basis of entougares of the diagonal all sets of the form

$$
V_{\epsilon}:=\left\{(\sigma, \eta) \in S_{\infty}: \varphi(\sigma, \eta)<\epsilon\right\},
$$

as $\epsilon$ runs over all positive reals. Equicontinuity of right translations means that for every $\epsilon>0$ there exists a $\delta>0$ such that whenever $(\sigma, \eta) \in V_{\delta}$ and $\theta \in S_{\infty}$, one has $(\sigma \theta, \eta \theta) \in V_{\epsilon}$.

Consider the following example. Let $n$ be even, and set

$$
\begin{aligned}
\sigma & =\left(\begin{array}{ccccccccc}
1 & 2 & 3 & 4 & 5 & 6 & \ldots & n-1 & n \\
2 & 1 & 4 & 3 & 6 & 5 & \ldots & n & n-1
\end{array}\right) \\
\eta & =\left(\begin{array}{ccccccccc}
1 & 2 & 3 & 4 & 5 & 6 & \ldots & n-1 & n \\
1 & 2 & 4 & 3 & 6 & 5 & \ldots & n & n-1
\end{array}\right) .
\end{aligned}
$$

One has $\varphi(\sigma, \eta)=2 / n$ and thus, by choosing $n$ sufficiently large, we can make the pair $(\sigma, \eta)$ belong to any entourage $V_{\delta}, \delta>0$. At the same time, $\varphi\left(\sigma \eta, \eta^{2}\right)=$ $\varphi\left(\left(\begin{array}{ll}1 & 2 \\ 2 & 1\end{array}\right), e\right)=2 / 2=1$, that is, the right translation of every entourage of the form $V_{\delta}$ is not a subset of $V_{1}$, however small $\delta>0$ be.

(Notice also that the same example works for left translations as well.) 